\title{
Sickle Hepatopathy
}

\author{
Manganas Konstantinos ${ }^{1 *}$, Delicou Sophia ${ }^{1}$ and Koskinas John ${ }^{2}$ \\ ${ }^{1}$ Thalassemia and Sickle Cell Department. Hippokration General Hospital Athens \\ ${ }^{2}$ Department of Medicine. National and Kapodistrian University of Athens, Medical School \\ Hippokration General Hospital
}

\section{ISSN: 2637-7632}

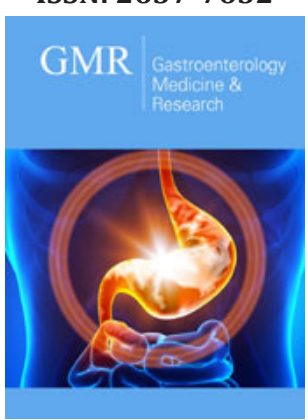

*Corresponding author: Manganas Konstantinos, Athens, Greece

Submission: 監 May 29, 2019

Published: 漹June 12, 2019

Volume 3 - Issue 3

How to cite this article: Manganas $\mathrm{K}$ Delicou S, Koskinas J.Sickle Hepatopathy. Gastro Med Res. 3(3). GMR.000561. 2019. DOI: 10.31031/GMR.2019.03.000561

Copyright@ Manganas Konstantinos,This article is distributed under the terms of the Creative Commons Attribution 4.0 International License, which permits unrestricted use and redistribution provided that the original author and source are credited.

\begin{abstract}
Liver involvement in sickle cell disease is multifactorial and is caused by either the vaso-occlusive phenomena that characterize the disease as well as hemolysis and transfusions or exchange transfusions that these patients often require and result in secondary liver hemochromatosis or transmission of infectious agents such as hepatitis B and C viruses. The acute and chronic liver complications of the disease are summarized under the term "sickle hepatopathy". The liver may be involved in sickle cell crisis with acute liver crisis, acute hepatic sequestration or acute intrahepatic cholestasis. Other acute manifestations associated with the disease are viral hepatitis, liver abscesses and occlusion of large vessels of the liver. Chronic liver involvement is mainly related to secondary hemochromatosis and chronic viral hepatitis that often lead to liver cirrhosis and liver failure if not treated, but also related with the increased formation of gallstones as well as chronic sickle cholangiopathy after repeated episodes of ischemic injury to the biliary tree.
\end{abstract}

\section{Mini Review}

Sickle cell disease (SCD) is one of the most common monogenic disorders worldwide. The replacement of glutamic acid by valine at position 6 of the $\beta$ globin molecule leads to the polymerization of hemoglobin $\mathrm{S}(\mathrm{HbS})$ in conditions of hypoxemia, with consequent intravascular hemolysis and vaso-occlusive effects. The disease affects a variety of organs of the human body, with the liver being one of the organs most frequently affected, both acutely and in chronic. The acute and chronic liver complications due to sickle cell disease are briefly described as "sickle hepatopathy" [1]. Sickle hepatopathy is primarily and predominantly associated with the pathophysiology of the disease itself, namely vaso-occlusive phenomena and hemolysis as a consequence of the sickling process, and secondary with the transfusion or exchange transfusion therapy that are often required in these patients.

\section{Acute Complications}

Liver involvement in sickle cell disease ranged from $10 \%$ to $39 \%[2,3]$ in several studies, while in another study, liver infarctions showed in 34\% of 70 patients with sickle cell disease [4]. There are no specific criteria for diagnosing liver involvement during sickle cell crises and clinical and laboratory findings are used. The clinical picture varies and ranges from simple liver biochemistry disorder or abdominal flatulence to intense right upper quadrant abdominal pain with painful hepatomegaly and rarely acute hepatic failure with encephalopathy and coagulation disorders. Hepatic injury is more commonly cholestatic or mixed type and rarely pure hepatocellular [3]. Laboratory testing reveals a varying degree of increase in transaminases, bilirubin and alkaline phosphatase. Intrahepatic sickling of the erythrocytes leads to obstruction of the liver sinusoids and the degree and severity of the sickling process and obstruction also determines the clinical manifestation. The general histological characteristics of liver disease due to sickle cell disease include obstruction, congestion and dilation of sinusoids, ischemic necrosis, Kuppfer cell hyperplasia, erythrophagocytosis and portal and perisinusoidal fibrosis following recurrent episodes of vaso-occlusions [4]. Histological findings are predominantly derived from mortuary material, since liver biopsy in sickle cell patients, even in a period outside the sickle cell crisis (steady state), is dangerous and can cause a crisis. Differential diagnosis of hepatic involvement in the crisis includes acute liver crisis, acute hepatic sequestration, acute intrahepatic cholestasis, acute viral hepatitis, cholecystitis and choledocholithiasis or cholangitis [1]. 


\section{Acute liver crisis}

The most common acute liver manifestation of sickle cell disease in which the sinusoid obstruction results in hepatic ischemia and infarction. Clinically, patients present with fever, jaundice, right upper quadrant abdominal pain and painful hepatomegaly, while a mild increase in transaminases and bilirubin is observed, whose values recede rapidly after the recession of the crisis, more usually in 3-14 days [5]. The prognosis for these patients is good and the treatment is supportive- hydration, oxygen therapy, analgesia and transfusions or exchange transfusions in more severe and persistent cases. It needs to be differentiated from acute cholecystitis or cholangitis with imaging methods and from acute viral hepatitis through serology testing.

\section{Acute hepatic sequestration}

This is another significant liver complication, although less common, with a mechanism like the sequestration of red cells in the spleen or lungs. Obstruction of the sinusoids and the entrapment of the red cells results in a significant distension of the sinusoids that cause compression and occlusion of intrahepatic bile vessels. Acute hepatic sequestration is clinically manifested by right upper quadrant abdominal pain, a significant degree of painless hepatomegaly and a significant decrease in hematocrit associated with the degree of hepatomegaly [6]. Patients may also experience the general symptoms of anemia with weakness and fatigue, but they may also present with the clinical picture of hypovolemic shock. As for the laboratory findings, transaminases are usually normal, since at least initially, there is no hepatocellular damage, while there is a significant increase in bilirubin, mainly its direct-conjugated fraction due to biliary obstruction, and an increase in alkaline phosphatase, often at three-digit values [5]. Treatment includes general supportive measures with hydration and maintenance of adequate oxygenation, as well as transfusion or exchange transfusion therapy for the treatment of anemia. Regular hematocrit control is required, as a rapid release of trapped nonhemolyzed erythrocytes into the circulation is often observed, with an increase in blood viscosity and risk for cardiovascular events and exchange transfusions are preferred to simple transfusions in this case [7].

\section{Acute intrahepatic cholestasis}

This is the most severe acute clinical manifestation of sickle cell disease concerning the liver and, although rarely, may be fatal. It is characterized by diffuse occlusion of liver sinusoids leading to ischemia, marked hepatocellular necrosis and intrahepatic cholestasis. It is initially presented as an acute liver crisis with fever and right upper quadrant abdominal pain and rapidly evolves into acute hepatic failure with coagulation disorders and hepatic encephalopathy. Frequently, renal insufficiency is also observed, probably due to renal infarctions or tubular necrosis, but with a mechanism not fully elucidated. Laboratory findings are characterized by a large increase in bilirubin often at threedigit values, mainly of its conjugated fraction, due to hemolysis, cholestasis and renal failure [8]. Transaminases also increase to three-digit values and PT, aPTT and INR are prolonged.
Treatment is, as in the previous cases, supportive with immediate onset of transfusions or exchange transfusions and administration of fresh frozen plasma for the treatment of coagulation disorders8. Renal function usually reverts after the treatment of liver insufficiency and usually chronic dialysis is not required, only temporary dialysis in the acute phase. The prognosis is poor. Out of 17 published cases of patients with acute intrahepatic cholestasis, 9 patients died [8,9]. Orthotropic liver transplantation for the treatment of acute intrahepatic cholestasis is being used in increasing frequency, but it is still controversial. There are 19 published cases of liver transplantation due to liver failure in sickle cell patients with a few transplantations due to acute intrahepatic cholestasis [10]. In a series of 6 cases of sickle cell patients who underwent urgent liver transplantation due to acute liver failure, the 3-year survival rate was $66.7 \%$ and the 10 year survival rate was $44.4 \%$ [11]. Patients followed a strict followup protocol before and after transplantation to keep hemoglobin $\mathrm{S}$ levels below $30 \%$. In this study the incidence of acute liver failure in sickle cell patients was $0.55 \%$ (11 out of 2000 patients). The main complications after transplantation were graft rejection $(2 / 6)$, neurological complications (4/6) and recurrence of pre-existing chronic hepatitis C (3/6). Vaso-occlusive liver crises continued to exist but were milder and less frequent.

\section{Liver abscess}

Rarely, fever and right upper quadrant abdominal pain can be due to the formation of liver abscesses, which are observed with an increased incidence in sickle cell disease due to functional asplenia. In this group of patients, abscesses from Yersinia enterocolitica are also formed more often than in general population due to secondary liver hemochromatosis and deferoxamine chelation therapy [12].

\section{Vascular complications}

Occasionally, cases of occlusion of the large vessels of the liver, such as hepatic or portal vein, have been reported. The clinical picture is usually not specific with abdominal pain that persists despite treatment and the diagnosis requires Doppler ultrasonography of the liver. Immediate administration of heparin or low molecular weight heparin is required, which can be changed to a vitamin $\mathrm{K}$ antagonist with a target INR of 2-3 for at least 6 months, if there is no malignancy or cirrhosis $[13,14]$.

\section{Chronic complications}

Chronicliver complicationsinclude secondaryhemochromatosis due to transfusion therapy, chronic hepatitis B and C and sickle cell cholangiopathy. Another common chronic complication of sickle cell disease is cholelithiasis and choledocholithiasis, while patients are often subjected to cholecystectomy, which is the most common surgical procedure in this group of patients, MRCP, ERCP or common bile duct investigation [5].

\section{Secondary hemochromatosis}

Frequent transfusions or exchange transfusions and chronic hemolysis that characterize the disease result in iron overload and secondary liver hemochromatosis. Iron is toxic to the liver and causes liver damage through formation of reactive oxygen 
species (ROS) and lipid peroxidation [15]. Initially, a mild hepatic biochemistry disorder is observed with hepatocellular damage and a transient increase in transaminases, but hepatic fibrosis and cirrhosis gradually develops due to chronic liver inflammation. At the same time, endocrine and cardiac disorders arise as a result of iron deposition in the pituitary gland and myocardium [15]. Indirect assessment, but not quantification, of liver iron can be achieved by measuring serum ferritin levels. A liver biopsy is considered the gold standard for assessment of hepatic iron concentration (HIC), with values greater than $15 \mathrm{mg} \mathrm{Fe} / \mathrm{g}$ dry liver tissue can be considered indicative of cirrhosis but poses risks to patients and has low repeatability. A reliable alternative test with good repeatability is MRI T2* of the liver, which offers a reliable liver iron concentration (LIC) measurement. Recently, liver elastography is used to assess liver fibrosis due to secondary hemochromatosis in sickle cell patients and liver stiffness values have been correlated with the liver iron concentration before and after chelation therapy [16]. The optimal cut-off value to diagnose liver cirrhosis is $>14 \mathrm{kPa}$ while values greater than $7 \mathrm{kPa}$ indicate liver fibrosis. On the other hand, values of liver stiffness lower than $7 \mathrm{kPa}$ does not exclude significant liver disease $[17,18]$. Patients with significant iron deposition require iron chelation therapy, with three agents available: deferoxamine, deferiprone and deferasirox. Deferasirox is a new oral iron chelator agent, more convenient for patients with an acceptable profile of safety and tolerability [19].

\section{Chronic viral hepatitis}

Another complication of transfusions, which is decreasing in recent years due to more effective blood donor control, is acute and chronic viral hepatitis B and C. In chronic viral hepatitis, patients are usually asymptomatic with a varying degree of transaminase elevation depending on activity, or are diagnosed with cirrhosis, the cause of which is found to be hepatitis B or C virus. The treatment of viral hepatitis in sickle cell disease follows the guidelines for the general population.

\section{Biliary complications}

Gallstone formation is observed with increased frequency in the disease as a result of increased bilirubin formation due to chronic hemolysis, so the stones are more commonly pigment gallstones. Patients with cholelithiasis remain asymptomatic for a long period of time with periodic episodes of abdominal pain after fatty meals, while choledocholithiasis, if it involves a significant obstruction of the common bile duct, results in right upper quadrant abdominal or epigastric pain and jaundice. Prophylactic cholecystectomy in asymptomatic patients with gallstone disease has been proposed by many authors but is still controversial [20].

Sickle cell cholangiopathy is due to ischemic damage to the biliary tree after repeated episodes of vaso-occlusions that result in fibrosis of intrahepatic or extrahepatic bile ducts. It is characterized by an increase, mainly of conjugated, bilirubin, alkaline phosphatase and a varying degree of transaminase elevation, while the MRCP initially shows dilatation of the bile ducts and in subsequent stages narrowing of the bile ducts due to fibrosis. In advanced stages of the disease the clinical picture of liver failure appears. Treatment is usually endoscopic in symptomatic patients.

\section{Conclusion}

Hepatopathy in sickle cell disease is an important factor in morbidity and mortality in this group of patients, both acutely and chronically. The term "sickle hepatopathy" describes the wide range of clinical manifestations of the disease concerning the liver and relates to vaso-occlusive effects, hemolysis and transfusions or exchange transfusions. The purpose of this review was to separate the clinical entities that are included in "sickle hepatopathy" according to their pathophysiology, which in many cases guides and their targeted treatment.

\section{References}

1. Banerjee S, Owen C, Chopra S (2001) Sickle cell hepatopathy Hepatology 33(5): 1021-1028.

2. Schubert TT (1986) Hepatobiliary system in sickle cell disease. Gastroenterology 90(6): 201-321.

3. Koskinas J, Manesis EK, Zacharakis GH, Galiatsatos N, Sevastos N, et al. (2007) Liver involvement in acute vaso-occlusive crisis of sickle cell disease: prevalence and predisposing factors. Scand J Gastroenterol 42(4): 499-507.

4. Bauer TW, Moore GW, Hutchins GM (1980) The liver in sickle cell disease: a clinicopathologic study of 70 patients. Am J Med 69(6): 833-837.

5. Shah R, Taborda C, Chawla S (2017) Acute and chronic hepatobiliary manifestations of sickle cell disease: A review. World J Gastrointest Pathophysiol 8(3): 108-116.

6. Hatton CS, Bunch C, Weatherall DJ (1985) Hepatic sequestration in sickle cell anaemia. Br Med J (Clin Res Ed) 290(6470): 744-745.

7. Lee ES, Chu PC (1996) Reverse sequestration in a case of sickle crisis. Postgrad Med J 72(850): 487-488.

8. Stéphan JL, Merpit-Gonon E, Richard O, Raynaud-Ravni C, Freycon F (1995) Fulminant liver failure in a 12-year-old girl with sickle cell anaemia: favourable outcome after exchange transfusions. Eur J Pediatr 154(6): 469-471.

9. Khan MA, Kerner JA (2011) Reversal of hepatic and renal failure from sickle cell intrahepatic cholestasis. Dig Dis Sci 56(6): 1634-1636.

10. Kwun Lui S, Krasinskas A, Shah R, Tracht JM (2019) Orthotropic liver transplantation for acute intrahepatic cholestasis in sickle cell disease: Clinical and histopathologic features of a rare case. Int J Surg Pathol 27(4): 411-417.

11. Hurtova M, Bachir D, Lee K, Calderaro J, Decaens T, et al. (2011) Transplantation for liver failure in patients with sickle cell disease: Challenging but feasible. Liver Transpl 17(4): 381-392.

12. Brunel F, François P, Durand C, Croize J, Jeannoel P (1993) Hepatic abscess due to Yersinia in a child with thalassemia. Pediatrie 48(2): 159162.

13. Sty RJ (1982) Ultrasonography: hepatic vein thrombosis in sickle cell anemia. Am J Pediatr Hematol Oncol 4(2): 213-215.

14. Dal Zotto C, Wieczorek M, Mariottini G, Malagò R, Olivieri O, et al. (2018) Persistent abdominal pain related to portal vein thrombosis in young adult with sickle cell disease. Am J Hematol 93(12): 1562-1565.

15. Raghupathy R, Manwani D, Little JA (2010) Iron overload in sickle cell disease. Adv Hematol 2010: 272940.

16. Delicou S, Maragkos K, Tambaki M, Kountouras D, Koskinas J (2018) Transient Elastography (TE) is a useful tool for assessing the response of liver iron chelation in sickle cell disease patients. Mediterr J Hematol Infect Dis 10(1): e2018049. 
17. Friedrich-Rust M, Ong MF, Martens S, Sarrazin C, Bojunga J, et al. (2008) Performance of transient elastography for the staging of liver fibrosis: a meta-analysis. Gastroenterology 134(4): 960-974.

18. Tsochatzis EA, Gurusamy KS, Ntaoula S, Cholongitas E, Davidson BR, et al. (2011) Elastography for the diagnosis of severity of fibrosis in chronic liver disease: a meta-analysis of diagnostic accuracy. J Hepatol 54(4): 650-659.
19. Ladis V, Drossou M, Vini D, Voskaridou E, Athanasiou-Metaxa M, et al. (2011) Deferasirox Safety Profile in Patients with TransfusionDependent Anaemias: Results From the Interim Analysis of a Hellenic Study. Blood 118(21): 3183.

20. Muroni M, Loi V, Lionnet F, Girot R, Houry S (2015) Prophylactic laparoscopic cholecystectomy in adult sickle cell disease patients with cholelithiasis: A prospective cohort study. Int J Surg 22: 62-66.

For possible submissions Click below: 